\title{
Unusual electrical behaviour in sol-gel-synthesised PKMO nano-sized manganite
}

\begin{abstract}
Mixed-valence manganites have gained tremendous attention in the scientific research for its colossal magnetoresistance phenomenon. Nevertheless, the study devoted to praseodymiumbased manganites is still limited to date. The present work aims to investigate the grain size effect on sol-gel grown nano-sized Pr0.85K0.15MnO3 (PKMO). The grain size has been modified by heat treatment ranging from $600{ }^{\circ} \mathrm{C}$ to $1000{ }^{\circ} \mathrm{C}$. PKMO samples have been studied in detail to elucidate the correlation of spin, charge, orbital and lattice degrees of freedom. The X-ray diffraction analysis revealed that all samples exhibit in single orthorhombic phase with the space group of Pnma (62). The obtained crystallite size and average grain size are in the range of $45-115 \mathrm{~nm}$ and $51-210 \mathrm{~nm}$, respectively. The evolution of grains intensively affects the electrical and magneto-transport properties of PKMO. The temperature dependence of the resistivity has been fitted with theoretical expressions in different temperature regimes to investigate their conduction mechanisms. The resistivity exhibits an unusual trend when the grain size increases where a similar pattern also been observed in metal-insulator transition temperature (TMI). This behaviour can be ascribed to the grain size distribution, grain formation and also the occurrence of oxygen vacancies at the grain boundaries. Enhancement of high field magnetoresistance has been discovered below $180 \mathrm{~K}$, whereas low field magnetoresistance is suppressed as the temperature increases and almost vanished at $300 \mathrm{~K}$. The PKMO study demonstrated here is clearly dominated by extrinsic properties (grain evolution) from the evidence of electrical and magneto-transport measurements.
\end{abstract}

